\title{
INTERTWINING FORMS FOR SUMMABLE INDUCED REPRESENTATIONS
}

\author{
BY \\ ADAM KLEPPNER(1)
}

1. Introduction. Calvin Moore [7] has recently introduced a variant of the notion of unitary induced representation for which he was able to give a version of the Frobenius Reciprocity Theorem which is an exact generalization of that theorem as it is known for finite groups. For finite groups the Reciprocity Theorem is a very particular case of results describing explictly the intertwining operators, or equivalently, the intertwining forms for two induced representations. In this paper we shall determine explicitly the intertwining forms for two induced representations of the kind introduced by Moore, which we shall call summable induced representations. The results parallel as closely as could reasonably be expected (that is, once all measure theoretic pathology has been ruled out) the results for finite groups. In particular, Moore's version of the Reciprocity Theorem is an immediate corollary.

To obtain these results we use methods due in their general outline to Bruhat [2], who employed them in his study of differentiable induced representations. The main idea is to describe the induced representation as a quotient of a representation which is essentially a regular representation acting on a space which is a tensor product. For the case of differentiable induced representations it is the nuclearity of the spaces involved which lies behind the success of the method. The summable induced representations act on spaces which are close to $L^{1}$ spaces. These spaces have some very special properties which serve as a substitute for nuclearity and which we now describe.

Let $X$ be a locally compact space and $\mu$ a positive (Radon) measure on $X$. Let $E$ be a Banach space and let $L^{1}(\mu, E)$ be the space of all classes of $\mu$-measurable functions $f: X \rightarrow E$ such that $\|f\|_{1}=\int\|f(x)\| d \mu(x)<\infty$. Provided with the indicated norm, $L^{1}(\mu, E)$ is a Banach space. The first important result concerning these spaces is the following theorem of Grothendieck [3, Chapitre I, Théorème 2].

THEOREM A. The map $f \otimes v \rightarrow f \cdot v, f \in L^{1}(\mu), v \in E$, where $f \cdot v(x)=f(x) v$ is an isometry of $L^{1}(\mu) \hat{\otimes} E$ with $L^{1}(\mu, E)$.

Received by the editors April 1, 1963.

(1) This work was partially supported by the National Science Foundation under Grant NSF-G25229. 
The analog of this result for spaces of distributions is the essence of Schwartz' Theorem of Kernels.

Let $L^{\infty}\left(\mu, E^{\prime}\right)$ be the space of all classes of $\mu$-measurable functions $f: X \rightarrow E^{\prime}$, where $E^{\prime}$ is provided with the weak topology $\sigma\left(E^{\prime}, E\right)$, which are essentially bounded ana set $\|f\|_{\infty}=$ ess sup $\|f(x)\|$. Then $L^{\infty}\left(\mu, E^{\prime}\right)$ is a Banach space.

Theorem B [1, Chapitre VI, §2, Proposition 10]. If $E$ is a separable Banach space the strong dual of $L^{1}(\mu, E)$ is isometric to $L^{\infty}\left(\mu, E^{\prime}\right)$ under the map which assigns to each $g \in L^{\infty}\left(\mu, E^{\prime}\right)$ the linear form

$$
f \rightarrow \int\langle g(x), f(x)\rangle d \mu(x) .
$$

This result is the analog of the very useful fact that the strong dual of the projective tensor product of two nuclear Fréchet spaces is the tensor product of their duals.

In the next section we give the definition of summable induced representations and recall certain other definitions. The third section is devoted to the determination of the intertwining forms and the fourth section is devoted to a discussion of intertwining numbers. The summable induced representations are closely related to unitary induced representations, and in the fifth section, making use of this relation, we give a lower bound for the intertwining number of two unitary induced representations. Unfortunately, to obtain this result we have to make the very restrictive assumption that the homogeneous spaces entering into play have finite invariant measures, and this restriction seriously limits the applicability of the result.

2. Summable induced representations. By a representation $L$ of the locally compact group $\mathscr{G}$ we will always mean a strongly continuous representation of $\mathscr{G}$ by isometries on a Banach space $\boldsymbol{H}(L)$. If $L$ is such a representation the subspace of all $v^{\prime} \in \boldsymbol{H}(L)^{\prime}$, such that $x \rightarrow{ }^{t} L_{x-1} v^{\prime}: \mathscr{G} \rightarrow \boldsymbol{H}(L)^{\prime}$ is continuous, is a strongly closed and weakly dense subspace $\boldsymbol{H}(\check{L})$ of $\boldsymbol{H}(L)^{\prime}$ (see [2, p. 113]). The map $x \rightarrow^{t} L_{x^{-1}} \mid \boldsymbol{H}(\breve{L})$ is a representation of $\mathscr{G}$ on $\boldsymbol{H}(\check{L})$ called the contragredient representation $\check{L}$ of $L$. If $G$ is a closed subgroup of $\mathscr{G}$ we denote by $L \mid G$ the restriction of $L$ to $G$.

Let $L$ and $M$ be two representations of $\mathscr{G}$. An intertwining form for $L$ and $M$ is a continuous bilinear form $B$ on $\boldsymbol{H}(L) \times \boldsymbol{H}(M)$ invariant under $L$ and $M$, that is, one satisfying

$$
B\left(L_{x} u, M_{x} v\right)=B(u, v), \quad x \in \mathscr{G}, \quad u, v \in \boldsymbol{H}(L) \times \boldsymbol{H}(M) .
$$

Let $B(L, M)$ denote the space of intertwining forms for $L$ and $M$. The space of all continuous bilinear forms on $\boldsymbol{H}(L) \times \boldsymbol{H}(M)$ is exactly the dual of $\boldsymbol{H}(L) \hat{\otimes} \boldsymbol{H}(M)$ (complete projective tensor product [3, Chapitre I, Définition 2]) which is a Banach 
space. We provide $\boldsymbol{B}(L, M)$ with the topology induced by the strong topology on $(\boldsymbol{H}(L) \hat{\otimes} \boldsymbol{H}(M))^{\prime}$. (As a general rule all duals will be equipped with the strong topology.) It is immediate that $\boldsymbol{B}(L, M)$ is a closed subspace, hence a Banach space. With this interpretation of bilinear forms, the intertwining forms are exactly those bilinear forms $B$ such that

$$
{ }^{t}\left(L_{x} \otimes M_{x}\right) B=B, \quad x \in \mathscr{G} .
$$

Each continuous bilinear form $B$ on $\boldsymbol{H}(L) \times \boldsymbol{H}(M)$ defines a continuous linear map $B^{\prime}: H(L) \rightarrow H(M)^{\prime}$ by setting $\left\langle B^{\prime} u, v\right\rangle=B(u, v)$. If $B \in B(L, M)$ then $B^{\prime}$ is an intertwining operator for the representations $L$ and $\check{M}$. Conversely, every intertwining operator for $L$ and $\check{M}$ is of the form $B^{\prime}$ and $B \rightarrow B^{\prime}$ is an isomorphism of $\boldsymbol{B}(L, M)$ on $\operatorname{Hom}_{\mathscr{G}}(L, \check{M})$, the space of intertwining operators.

We shall denote by $\mu$ or $d_{\mathscr{G}}(x)$ a right invariant Haar measure on $\mathscr{G}$ and by $\Delta$ the modular function of $\mathscr{G}$, that is, the function such that

$$
\Delta(x) \int f(x y) d_{\mathscr{g}}(y)=\int f(y) d_{\mathscr{g}}(y), \quad x \in \mathscr{G} .
$$

If $G$ is a closed subgroup of $\mathscr{G}$ we denote by $d_{G}(x)$ a right invariant Haar measure on $G$ and by $\delta_{G}$, or $\delta$, the modular function of $G$.

Let $\rho_{G}$, or $\rho$, be a continuous positive function on $\mathscr{G}$ such that

$$
\rho(e)=1, \quad \rho(\xi x)=\delta(\xi) \Delta(\xi)^{-1} \rho(x), \quad \xi, x \in G \times \mathscr{G} .
$$

The existence of such functions, called $\rho$-functions, results, for the case of separable Lie groups, from a construction due to Bruhat [2]. However, his construction can be carried through without change for arbitrary locally compact groups once it has been noted that the homogeneous space $\mathscr{G} / G$ of right $G$-cosets is paracompact. To this function $\rho$ is associated a quasi-invariant measure $v$ on $\mathscr{G} / G$ defined by the equation

$$
\int_{\mathscr{G}} f(x) \rho(x) d_{\mathscr{G}}(x)=\int_{\mathscr{G} / G} \int_{G} f(\xi x) d_{G}(\xi) d v(\bar{x}),
$$

where $f$ is a continuous function with compact support on $\mathscr{G}, \bar{x}$ is the image of $x$ in $\mathscr{G} / G$, and the meaning of the expression on the right lies in the fact that $\int f(\xi x) d_{G}(\xi)$ depends only on $\bar{x}$ and may be denoted by $\bar{f}(\bar{x})$. Thus the measure $v$ is defined by

$$
\int_{\mathscr{G} / G} \bar{f}(\bar{x}) d v(\bar{x})=\int_{\mathscr{G}} f(x) \rho(x) d_{\mathscr{G}}(x) .
$$

For each $s \in \mathscr{G}$ the function $x \rightarrow \rho(x s) / \rho(x)$ depends only on $\bar{x}$ and the quasiinvariance of $v$ is expressed by the equation:

$$
\int_{\mathscr{g} / G} \bar{f}(\overline{x s}) \frac{\rho(x s)}{\rho(x)} d v(\bar{x})=\int_{\mathscr{G} / G} \bar{f}(\bar{x}) d v(\bar{x}) .
$$


For further details see [4].

Let $L$ be a representation of the closed subgroup $G$ of the locally compact group $\mathscr{G}$ and let $\boldsymbol{H}\left(V^{L}\right)$ be the set of all (classes of ) measurable (meaning always, Haar measurable) functions $f: \mathscr{G} \rightarrow \boldsymbol{H}(L)$ such that:

(a) $f(\xi x)=L_{\xi} f(x), \xi, x \in G \times \mathscr{G}$,

(b) $\|f\|=\int_{\mathscr{g} / \mathrm{G}}\|f(x)\| d v(\bar{x})<\infty$.

The integral in (b) makes sense since by (a) the integrand $x \rightarrow\|f(x)\|$ depends only on $\bar{x}$. Provided with the indicated norm, $\boldsymbol{H}\left(V^{L}\right)$ is a normed space. It is easy to verify, if there exists a Borel cross section of $\mathscr{G} / G$ in $\mathscr{G}$, that $H\left(V^{L}\right)$ is isometric to $L^{1}(v, \boldsymbol{H}(L))$ and is accordingly a Banach space. In particular, this is the case if $\mathscr{G}$ is separable [4, Lemma 1.1$]$.

For each $s \in \mathscr{G}$ and $f \in \boldsymbol{H}\left(V^{L}\right)$ define

$$
V_{s}^{L} f(x)=\frac{\rho(x s)}{\rho(x)} f(x s) .
$$

Then $V_{s}^{L} f(\xi x)=L_{\xi} V_{s}^{L} f(x), \xi \in G$, and

$$
\left\|V_{s}^{L} f\right\|=\int_{\mathscr{G} / G} \frac{\rho(x s)}{\rho(x)}\|f(x s)\| d v(\bar{x})=\int_{\mathscr{g} / G}\|f(x)\| d v(\bar{x})=\|f\| .
$$

Thus each $V_{s}^{L}$ is an isometry of $\boldsymbol{H}\left(V^{L}\right)$; moreover, $s \rightarrow V_{s}^{L}$ is a homomorphism of $\mathscr{G}$ into the group of isometries of $\boldsymbol{H}\left(V^{L}\right)$. It will follow from Lemmas 1 and 2 below, when there exists a Borel cross section of $\mathscr{G} / G$ in $\mathscr{G}$ and $G$ is $\sigma$-compact, that the map $s \rightarrow V_{s}^{L}$ is strongly continuous. In this case $H\left(V^{L}\right)$ is a Banach space and $V^{L}$ is a representation of $\mathscr{G}$ which we shall call the summable representation induced from $L$, to distinguish it from the unitary induced representations of Mackey which may be thought of as a particular kind of square summable induced representation. More generally, whenever $\boldsymbol{H}\left(V^{L}\right)$ is complete and $s \rightarrow V_{s}^{L}$ is strongly continuous, which is probably always the case, we shall call $V^{L}$ the summable representation induced from $L$. This notion of summable induced representation is due to C. Moore [7].

A summable induced representation is defined as the restriction to an invariant subspace of a certain representation, closely related to a regular representation. Following the general pattern outlined by Bruhat [2] for differentiable representations, we shall describe each summable induced representation as a quotient of a representation acting on a tensor product. For this, let $\mathscr{K}(\mathscr{G}, \boldsymbol{H}(L))$ be the space of all continuous functions with compact support on $\mathscr{G}$ and values in $\boldsymbol{H}(L)$. We define a map $\pi: \mathscr{K}(\mathscr{G}, \boldsymbol{H}(L)) \rightarrow \boldsymbol{H}\left(V^{L}\right)$ by setting

$$
\pi f(x)=\int_{G} L_{\xi}^{-1} f(\xi x) d_{G}(\xi)
$$

where the integral is the weak integral. For $\eta \in G$ we have 


$$
\begin{aligned}
\pi f(\eta x) & =\int_{G} L_{\xi}^{-1} f(\xi \eta x) d_{G}(\xi)=\int_{G} L_{\eta} L_{\xi \eta}^{-1} f(\xi \eta x) d_{G}(\xi) \\
& =L_{\eta} \int_{G} L_{\xi}^{-1} f(\xi x) d_{G}(\xi)=L_{\eta} \pi f(x) .
\end{aligned}
$$

Also

$$
\|\pi f(x)\| \leqq \int_{G}\left\|L_{\xi}^{-1} f(\xi x)\right\| d_{G}(\xi)=\int_{G}\|f(\xi x)\| d_{G}(\xi)
$$

and from this follows the inequality

$$
\begin{aligned}
\int_{\mathscr{g} / G}\|\pi f(x)\| d v(\bar{x}) & \leqq \int_{\mathscr{G} / G} \int_{G}\|f(\xi x)\| d_{G}(\xi) d v(\bar{x}) \\
& =\int_{\mathscr{G}}\|f(x)\| \rho(x) d_{\mathscr{G}}(x)<\infty .
\end{aligned}
$$

By (2) and (3), $\pi f \in \boldsymbol{H}\left(V^{L}\right)$. Moreover, (2) also states that $\|\pi f\| \leqq\|f\|_{1}$, where \|\|$_{1}$ is the norm in $L^{1}(\rho \mu, H(L))$. Thus $\pi$ can be extended to a norm decreasing linear map of $L^{1}(\rho \mu, \boldsymbol{H}(L))$ into the completion of $\boldsymbol{H}\left(V^{L}\right)$. e denote this extension by $\pi_{L}$, or $\pi$.

LEMmA 1. If there exists a Borel cross section $\theta: \mathscr{G} / G \rightarrow \mathscr{G}$ and if $G$ is $\sigma$ compact, then $\pi_{L}$ is a homomorphism of $L^{1}(\rho \mu, H(L))$ on $\boldsymbol{H}\left(V^{L}\right)$ and $\left\|\pi_{L}\right\|=1$.

Proof. Let $\alpha$ be a continuous positive function on $G$ such that $\int_{G} \alpha(\xi) d_{G}(\xi)=1$. For $f \in \boldsymbol{H}\left(V^{L}\right)$ set $p f(x)=\alpha(\phi x) L_{\phi x} f(\theta \bar{x})$, where $\phi(x)=x(\theta \bar{x})^{-1}$. Then $p f: \mathscr{G} \rightarrow \boldsymbol{H}(L)$ is measurable and

$$
\begin{aligned}
\|p f\|_{1} & =\int_{\mathscr{S}}\|p f(x)\| \rho(x) d_{\mathscr{G}}(x)=\int_{\mathscr{G} / G} \int_{G}\|p f(\xi x)\| d_{G}(\xi) d v(\bar{x}) \\
& =\int_{\mathscr{S} / G} \int_{G} \alpha(\xi \phi x)\|f(\theta \bar{x})\| d_{\mathscr{G}}(\xi) d v(\bar{x}) \\
& =\int_{\mathscr{S} / G}\|f(\theta \bar{x})\| \int_{G} \alpha(\xi \phi x) d_{G}(\xi) d v(\bar{x})=\int_{\mathscr{G} / G}\|f(\theta \bar{x})\| d v(\bar{x}) \\
& =\|f\| .
\end{aligned}
$$

Thus $p f \in L^{1}(\rho \mu, \boldsymbol{H}(L))$ and $p: \boldsymbol{H}\left(V^{L}\right) \rightarrow L^{1}(\rho \mu, \boldsymbol{H}(L))$ is an isometry. Because

$$
L_{\xi}^{-1} p f(\xi x)=L_{\xi}^{-1} \alpha(\xi \phi x) L_{\xi \phi x} f(\theta \bar{x})=\alpha(\xi \phi x) L_{\phi x} f(\theta \bar{x})
$$

we have

$$
\begin{aligned}
\pi p f(x) & =\int_{G} L_{\xi}^{-1} p f(\xi x) d_{G}(\xi)=\int_{G} \alpha(\xi \phi x) L_{\phi x} f(\theta \bar{x}) d_{G}(\xi) \\
& =L_{\phi x} f(\theta \bar{x})=f(\phi x(\theta \bar{x}))=f(x) .
\end{aligned}
$$


Thus $\pi p f=f$ and $p$ is a right inverse for $\pi$. Because $H\left(V^{L}\right)$ is a Banach space, $\pi L^{1}(\rho \mu, \boldsymbol{H}(L)) \subset \boldsymbol{H}\left(V^{L}\right)$ and because $p$ is a right inverse for $\pi, \pi$ is surjective, and by the open mapping theorem $\pi$ is a homomorphism. We have already seen that $\pi$ has norm no greater than 1 , and $\pi p f=f$ implies that $\pi$ has norm exactly equal to 1 .

For each $s \in \mathscr{G}$ and $f \in L^{1}(\rho \mu, \boldsymbol{H}(L))$ set

$$
\tau_{s}^{L} f(x)=\frac{\rho(x s)}{\rho(x)} f(x s)
$$

LeMma 2. $\tau^{L}: s \rightarrow \tau_{s}^{L}$ is a representation of $\mathscr{G}$ on $L^{1}(\rho \mu, H(L))$ and $\pi_{L} \tau_{s}^{L}=V_{s}^{L} \pi_{L}$, all $s \in \mathscr{G}$.

Proof. For each $s \in \mathscr{G}$,

$$
\begin{aligned}
\left\|\tau_{s}^{L} f\right\|_{1} & =\int_{\mathscr{G}}\left\|\tau_{s}^{L} f(x)\right\| \rho(x) d_{\mathscr{G}}(x)=\int_{\mathscr{G}} \rho(x s)\|f(x s)\| d_{\mathscr{G}}(x) \\
& =\int_{\mathscr{G}} \rho(x)\|f(x)\| d_{\mathscr{G}}(x)=\|f\|_{1} .
\end{aligned}
$$

Thus each $\tau_{s}^{L}$ is an isometry. For $s, t$ in $\mathscr{G}$,

$$
\tau_{s}^{L} \tau_{t}^{L} f(x)=\frac{\rho(x t)}{\rho(x)} \tau_{s}^{L} f(x t)=\frac{\rho(x t)}{\rho(x)} \frac{\rho(x t s)}{\rho(x t)} f(x t s)=\tau_{t s}^{L} f(x)
$$

and $\tau^{L}$ is a homomorphism of $\mathscr{G}$ into the group of isometries of $L^{1}(\rho \mu, \boldsymbol{H}(L))$. Because the map $f \rightarrow \rho f$ is an isometry of $L^{1}(\rho \mu, \boldsymbol{H}(L))$ with $L^{1}(\mu, \boldsymbol{H}(L))$ and under this isometry $\tau^{L}$ corresponds to the right regular representation of $\mathscr{G}$ on $L^{1}(\mu, H(L))$ which is strongly continuous, $\tau^{L}$ is itself strongly continuous, thus a representation of $\mathscr{G}$. Finally, we have

$$
\begin{aligned}
\pi_{L} \tau_{s}^{L} f(x) & =\int_{G} L_{\xi}^{-1} \tau_{s}^{L} f(\xi x) d_{G}(\xi)=\int_{G} L_{\xi}^{-1} \frac{\rho(x s)}{\rho(x)} f(\xi x s) d_{G}(\xi) \\
& =V_{s}^{L} \pi_{L} f(x)
\end{aligned}
$$

and $\pi_{L}$ intertwines the representations $\tau^{L}$ and $V^{L}$.

This is the desired presentation of $V^{L}$ as a quotient representation. A corollary of this result is the fact that under the conditions of Lemma $1 V^{L}$ is strongly continuous. Indeed, this follows immediately from the strong continuity of $\tau^{L}$ and the surjectivity of $\pi_{L}$.

We shall ordinarily write $\tau$ for $\tau^{L}$.

3. Intertwining forms. Let $G_{1}$ and $G_{2}$ be closed $\sigma$-compact subgroups of the locally compact $\mathscr{G}$ and suppose there exists a Borel cross section of $\mathscr{G} / G_{i}$ in $\mathscr{G}$, $i=1,2$. Choose finite quasi-invariant measures $v_{i}$ on $\mathscr{G} / G_{i}$ corresponding to continuous $\rho$ functions $\rho_{i}, i=1,2$. Let $L$ and $M$ be representations of $G_{1}$ and $G_{2}$ respectively for which $\boldsymbol{H}(L)$ and $\boldsymbol{H}(M)$ are separable. 
Suppose $B$ is an intertwining form for $V^{L}$ and $V^{M}$. Then $B^{\prime}(f, g)=B\left(\pi_{L} f, \pi_{M} g\right)$ is a continuous bilinear form on $L^{1}\left(\rho_{1} \mu, \boldsymbol{H}(L)\right) \times L^{1}\left(\rho_{2} \mu, \boldsymbol{H}(M)\right)$, that is,

$$
B^{\prime} \in\left(L^{1}\left(\rho_{1} \mu, \boldsymbol{H}(L)\right) \hat{\otimes} L^{1}\left(\rho_{2} \mu, \boldsymbol{H}(M)\right)\right)^{\prime} .
$$

Moreover, $B \rightarrow B^{\prime}$ is the map ${ }^{t}\left(\pi_{L} \otimes \pi_{M}\right)$ and because $\pi_{L}$ and $\pi_{M}$ are homomorphisms of norm 1 (Lemma 1 ), $\pi_{L} \otimes \pi_{M}$ is a homomorphism [3, Chapitre I, Proposition 3]. Moreover, it follows from the proof of Lemma 1 that $\operatorname{ker} \pi_{L}$ and $\operatorname{ker} \pi_{M}$ are direct factors which implies that $\pi_{L} \otimes \pi_{M}$ has norm equal to 1 . Thus ${ }^{t}\left(\pi_{L} \otimes \pi_{M}\right)$ is an isomorphism of norm 1 and in fact an isometry.

By Theorem A [3, Chapitre I, Théorème 2], $L^{1}\left(\rho_{1} \mu, \boldsymbol{H}(L)\right)=L^{1}\left(\rho_{1} \mu\right) \hat{\otimes} \boldsymbol{H}(L)$ and $L^{1}\left(\rho_{2} \mu, \boldsymbol{H}(M)\right)=L^{1}\left(\rho_{2} \mu\right) \hat{\otimes} \boldsymbol{H}(M)$. Thus

$$
L^{1}\left(\rho_{1} \mu, \boldsymbol{H}(L)\right) \hat{\otimes} L^{1}\left(\rho_{2} \mu, \boldsymbol{H}(M)\right)=L^{1}\left(\rho_{1} \mu\right) \hat{\otimes} \boldsymbol{H}(L) \hat{\otimes} L^{1}\left(\rho_{2} \mu\right) \hat{\otimes} \boldsymbol{H}(M) .
$$

But by the commutativity of the tensor product and two more applications of Theorem A,

$$
\begin{aligned}
L^{1}\left(\rho_{1} \mu, \boldsymbol{H}(L)\right) \hat{\otimes} L^{1}\left(\rho_{2} \mu, \boldsymbol{H}(M)\right) & =L^{1}\left(\rho_{1} \mu\right) \hat{\otimes} L^{1}\left(\rho_{2} \mu\right) \hat{\otimes} \boldsymbol{H}(L) \hat{\otimes} \boldsymbol{H}(M) \\
& =L^{1}\left(\rho_{1} \mu \otimes \rho_{2} \mu\right) \hat{\otimes} \boldsymbol{H}(L) \hat{\otimes} \boldsymbol{H}(M) \\
& =L^{1}\left(\rho_{1} \mu \otimes \rho_{2} \mu, \boldsymbol{H}(L) \hat{\otimes} \boldsymbol{H}(M)\right) .
\end{aligned}
$$

Because $\boldsymbol{H}(L)$ and $\boldsymbol{H}(M)$ are separable, so is $\boldsymbol{H}(L) \hat{\otimes} \boldsymbol{H}(M)$ and by Theorem B [1, Chapitre VI, §2, Proposition 10]

$$
\left(L^{1}\left(\rho_{1} \mu \otimes \rho_{2} \mu, \boldsymbol{H}(L) \hat{\otimes} \boldsymbol{H}(M)\right)\right)^{\prime}
$$

is isometric to

$$
L^{\infty}\left(\rho_{1} \mu \otimes \rho_{2} \mu,(\boldsymbol{H}(L) \hat{\otimes} \boldsymbol{H}(M))^{\prime}\right)=\boldsymbol{E}(L, M) .
$$

Explicitly this isometry is the map which assigns to each $\beta \in E(L, M)$ (more exactly, to the class containing $\beta$ ) the bilinear form

$$
B: f, g \rightarrow \int_{\mathscr{G} \times \mathscr{G}}\langle\beta(x, y), f(x) \otimes g(y)\rangle \rho_{1}(x) \rho_{2}(y) d_{\mathscr{G} \times \mathscr{G}}(x, y)
$$

for $f \otimes g \in L^{1}\left(\rho_{1} \mu \otimes \rho_{2} \mu, \boldsymbol{H}(L) \hat{\otimes} \boldsymbol{H}(M)\right)$. Composing this isometry with the isometry ${ }^{t}\left(\pi_{L} \otimes \pi_{M}\right)$ gives an isometry of $B(L, M)$ with a closed subspace of $\boldsymbol{E}(L, M)$, which we shall describe exactly.

Let $B$ be an intertwining form for $V^{L}$ and $V^{M}$ and set $B^{\prime}={ }^{t}\left(\pi_{L} \otimes \pi_{M}\right) B$. Let $\beta \in \boldsymbol{E}(L, M)$ be the function such that

$$
B^{\prime}(f, g)=\int_{\mathscr{G} \times \mathscr{G}}\langle\beta(x, y), f(x) \otimes g(y)\rangle \rho_{1}(x) \rho_{2}(y) d_{\mathscr{G} \times \mathscr{G}}(x, y) .
$$

Because 


$$
\begin{aligned}
B^{\prime}\left(\tau_{s} f, \tau_{s} g\right) & =B\left(\pi_{L} \tau_{s} f, \pi_{M} \tau_{s} g\right)=B\left(V_{s}^{L} \pi_{L} f, V_{s}^{M} \pi_{M} g\right)=B\left(\pi_{L} f, \pi_{M} g\right) \\
& =B^{\prime}(f, g),
\end{aligned}
$$

where we have written $\tau$ for both $\tau^{L}$ and $\tau^{M}$, we have

$$
\begin{aligned}
B^{\prime}\left(\tau_{s} f, \tau_{s} g\right) & =\int_{\mathscr{G} \times \mathscr{G}}\langle\beta(x, y), f(x s) \otimes g(y s)\rangle \frac{\rho_{1}(x s)}{\rho_{1}(x)} \frac{\rho_{2}(y s)}{\rho_{2}(y)} \rho_{1}(x) \rho_{2}(y) d_{\mathscr{G} \times \mathscr{G}}(x, y) \\
& =\int_{\mathscr{G} \times \mathscr{G}}\langle\beta(x, y), f(x s) \otimes g(y s)\rangle \rho_{1}(x s) \rho_{2}(y s) d_{\mathscr{G} \times \mathscr{G}}(x, y) \\
& =\int_{\mathscr{G} \times \mathscr{G}}\left\langle\beta\left(x s^{-1}, y s^{-1}\right), f(x) \otimes g(y)\right\rangle \rho_{1}(x) \rho_{2}(y) d_{\mathscr{g} \times \mathscr{g}}(x, y) \\
& =\int_{\mathscr{g} \times \mathscr{G}}\langle\beta(x, y), f(x) \otimes g(y)\rangle \rho_{1}(x) \rho_{2}(y) d_{\mathscr{G} \times \mathscr{G}}(x, y)=B^{\prime}(f, g) .
\end{aligned}
$$

Because $f$ and $g$ are arbitrary it must be that for each $s \in \mathscr{G}$

$$
\beta\left(x s^{-1}, y s^{-1}\right)=\beta(x, y), \quad \text { almost all } x, y \in \mathscr{G} \times \mathscr{G},
$$

that is, the equality holds for all $x, y$ not in a set of Haar measure 0 .

To study the situation more closely we define for each $\eta \in G_{1}$ an operator $\sigma_{\eta}^{L}=\sigma_{\eta}$ on $L^{1}\left(\rho_{1} \mu, \boldsymbol{H}(L)\right)$ by:

$$
\sigma_{\eta} f(x)=\delta_{1}(\eta) f(\eta x)
$$

where $\delta_{1}=\delta_{G_{1}}$ is the modular function of $G_{1}$. Then

$$
\begin{aligned}
\left\|\sigma_{\eta} f\right\|_{1} & =\int_{\mathscr{G}}\left\|\sigma_{\eta} f(x)\right\| \rho_{1}(x) d_{\mathscr{G}}(x)=\delta_{1}(\eta) \int_{\mathscr{G}}\|f(\eta x)\| \rho_{1}(x) d_{\mathscr{g}}(x) \\
& =\Delta(\eta)^{-1} \delta_{1}(\eta) \int_{\mathscr{G}}\|f(x)\| \rho_{1}\left(\eta^{-1} x\right) d_{\mathscr{G}}(x) .
\end{aligned}
$$

By the properties (1) of the function $\rho_{1}$

$$
\Delta(\eta)^{-1} \delta_{1}(\eta) \rho_{1}\left(\eta^{-1} x\right)=\rho(x)
$$

so that

$$
\left\|\sigma_{\eta} f\right\|_{1}=\int_{\mathscr{G}}\|f(x)\| \rho(x) d_{\mathscr{G}}(x)=\|f\|_{1}
$$

Thus each $\sigma_{\eta}$ is an isometry, and $\sigma$ is a strongly continuous anti-representation of $G_{1}$ in $L^{1}\left(\rho_{1} \mu, H(L)\right)$. Further, $\sigma$ has the following property: 


$$
\begin{aligned}
\pi_{L}\left(\sigma_{\eta} f\right)(x) & =\int_{G_{1}} L_{\xi}^{-1} \delta_{1}(\eta) f(\eta \xi x) d_{G_{1}}(\xi) \\
& =\delta_{1}(\eta) \int_{G_{1}} L_{\eta \xi}^{-1} L_{\eta} f(\eta \xi x) d_{G_{1}}(\xi)=\int_{G_{1}} L_{\xi}^{-1} L_{\eta} f(\xi x) d_{G_{1}}(\xi) \\
& =\pi_{L}\left(L_{\eta} f\right)(x) .
\end{aligned}
$$

In a similar manner we define an anti-representation $\sigma=\sigma^{M}$ of $G_{2}$ on $L^{1}\left(\rho_{2} \mu, H(M)\right)$.

Again let $B$ be an intertwining form for $V^{L}$ and $V^{M}$ and set $B^{\prime}={ }^{t}\left(\pi_{L} \otimes \pi_{M}\right) B$. Let $\beta$ be the function in $E(L, M)$ which defines $B^{\prime}$ as in (4). For all $\eta, \zeta \in G_{1} \times G_{2}$

$$
\begin{aligned}
B^{\prime}\left(\sigma_{\eta} f, \sigma_{\zeta} g\right) & =B\left(\pi_{L} \sigma_{\eta} f, \pi_{M} \sigma_{\zeta} g\right)=B\left(\pi_{L} L_{\eta} f, \pi_{M} M_{\zeta} g\right)=B^{\prime}\left(L_{\eta} f, M_{\zeta} g\right) \\
& =\int_{\mathscr{S} \times \mathscr{g}}\left\langle\beta(x, y), L_{\eta} f(x) \otimes M_{\zeta} g(y)\right\rangle \rho_{1}(x) \rho_{2}(y) d_{\mathscr{G} \times \mathscr{G}}(x, y) \\
& =\int_{\mathscr{G} \times \mathscr{G}}\left\langle{ }^{t}\left(L_{\eta} \otimes M_{\zeta}\right) \beta(x, y), f(x) \otimes g(y)\right\rangle \rho_{1}(x) \rho_{2}(y) d_{\mathscr{G} \times \mathscr{g}}(x, y) .
\end{aligned}
$$

On the other hand,

$$
\begin{aligned}
B^{\prime}\left(\sigma_{\eta} f, \sigma_{\zeta} g\right) & =\int_{\mathscr{g} \times \mathscr{G}}\left\langle\beta(x, y), \sigma_{\eta} f(x) \otimes \sigma_{\zeta} g(y)\right\rangle \rho_{1}(x) \rho_{2}(y) d_{\mathscr{g} \times \mathscr{G}}(x, y) \\
& =\delta_{1}(\eta) \delta_{2}(\zeta) \int_{\mathscr{S} \times \mathscr{G}}\left\langle\beta(x, y), f(\eta x) \otimes g(\zeta y) \rho_{1}(x) \rho_{2}(y) d_{\mathscr{g} \times \mathscr{g}}(x, y)\right. \\
& =\delta_{1}(\eta) \delta_{2}(\zeta) \Delta(\eta)^{-1} \Delta(\zeta)^{-1} \\
& \times \int_{\mathscr{g} \times \mathscr{g}}\left\langle\beta\left(\eta^{-1} x, \zeta^{-1} y\right), f(x) \otimes g(y)\right\rangle \rho_{1}\left(\eta^{-1} x\right) \rho_{2}\left(\zeta^{-1} y\right) d_{\mathscr{g} \times \mathscr{g}}(x, y) \\
& =\int_{\mathscr{S} \times \mathscr{g}}\left\langle\beta\left(\eta^{-1} x, \zeta^{-1} y\right), f(x) \otimes g(y)\right\rangle \rho_{1}(x) \rho_{2}(y) d_{\mathscr{G} \times \mathscr{G}}(x, y),
\end{aligned}
$$

since $\delta_{1}(\eta) \Delta(\eta)^{-1} \rho_{1}\left(\eta^{-1} x\right)=\rho_{1}(x)$, and similarly for $\rho_{2}$. Comparing (7) and (8) we find, for all $\eta, \zeta \in G_{1} \times G_{2}$,

$$
\beta\left(\eta^{-1} x, \zeta^{-1} y\right)={ }^{t}\left(L_{\eta} \otimes M_{\zeta}\right) \beta(x, y), \quad \text { almost all } x, y \in \mathscr{G} \times \mathscr{G} .
$$

These two properties (5) and (9) completely characterize the subspace of $E(L, M)$ which we were seeking.

TheOREM 1. Let $G_{1}$ and $G_{2}$ be closed $\sigma$-compact subgroups of the locally compact group $\mathscr{G}$ and suppose there exists a Borel cross section of $\mathscr{G} / G_{i}$ in $\mathscr{G}$, $i=1,2$. Let $v_{i}$ be a quasi-invariant measure on $\mathscr{G} / G_{i}$ associated to the continuous $\rho$ function $\rho_{i}, i=1,2$. Let $L$ and $M$ be strongly continuous representations by isometries on separable Banach spaces $H(L)$ and $H(M)$ of $G_{1}$ and $G_{2}$, respectively. 
Let $\boldsymbol{F}(L, M)$ be the subspace of all $\beta \in L^{\infty}\left(\rho_{1} \mu \otimes \rho_{2} \mu,(\boldsymbol{H}(L) \hat{\otimes} \boldsymbol{H}(M))^{\prime}\right)$ which satisfy, for all $\eta, \zeta, s \in G_{1} \times G_{2} \times \mathscr{G}$

(10) $\quad \beta\left(\eta^{-1} x s, \zeta^{-1} y s\right)={ }^{t}\left(L_{\eta} \otimes M_{\zeta}\right) \beta(x, y), \quad$ almost all $x, y \in \mathscr{G} \times \mathscr{G}$.

For each $\beta \in \boldsymbol{F}(L, M)$ and $f \otimes g \in \boldsymbol{H}\left(V^{L}\right) \otimes \boldsymbol{H}\left(V^{M}\right)$, the function

$$
x, y \rightarrow\langle\beta(x, y), f(x) \otimes g(y)\rangle
$$

depends only on the $G_{1} \times G_{2}$ coset to which $x, y$ belongs, and

$$
B: f, g \rightarrow \int_{\mathscr{G} / G_{1} \times g / G_{2}}\langle\beta(x, y), f(x) \otimes g(y)\rangle d\left(v_{1} \otimes v_{2}\right)(\bar{x}, \bar{y})
$$

is an intertwining form for $V^{L}$ and $V^{M}$. The map $\beta \rightarrow B$ is a linear isometry of $\boldsymbol{F}(L, M)$ on $\boldsymbol{B}(L, M)$.

Proof. We have already seen that to each intertwining form $B$ there corresponds $\beta \in F(L, M)$, and $B \rightarrow \beta$ is an isometry. Moreover, if $\beta \in F(L, M)$ for $f \otimes g \in \boldsymbol{H}\left(V^{L}\right) \otimes \boldsymbol{H}\left(V^{M}\right)$ and $\eta, \zeta \in G_{1} \times G_{2}$,

$$
\begin{aligned}
\langle\beta(\eta x, \zeta y), f(\eta x) \otimes g(\zeta y)\rangle & =\left\langle{ }^{t}\left(L_{\eta}^{-1} \otimes M_{\zeta}^{-1}\right) \beta(x, y), L_{\eta} \otimes M_{\zeta} f(x) \otimes g(y)\right\rangle \\
& =\langle\beta(x, y), f(x) \otimes g(y)\rangle
\end{aligned}
$$

so that $x, y \rightarrow\langle\beta(x, y), f(x) \otimes g(y)\rangle$ depends only on the $G_{1} \times G_{2}$ coset to which $x, y$ belongs.

Choose $\beta \in F(L, M)$ and let $B^{\prime}$ be the bilinear form on

$$
L^{1}\left(\rho_{1} \mu, H(L)\right) \times L^{1}\left(\rho_{2} \mu, H(M)\right)
$$

defined by $\beta$, that is,

Then

$$
B^{\prime}(f, g)=\int_{\mathscr{G} \times \mathscr{G}}\langle\beta(x, y), f(x) \otimes g(y)\rangle \rho_{1}(x) \rho_{2}(y) d_{\mathscr{g} \times \mathscr{g}}(x, y) .
$$

$$
\begin{aligned}
B^{\prime}(f, g)= & \int_{\mathscr{S} \times \mathscr{S} / G_{1} \times G_{2}} \int_{G_{1} \times G_{2}}\langle\beta(\eta x, \zeta y), f(\eta x) \otimes g(\zeta y)\rangle d_{G_{1} \times G_{2}}(\eta, \zeta) d\left(v_{1} \otimes v_{2}\right)(\bar{x}, \bar{y}) \\
= & \int_{\mathscr{g} \times \mathscr{S} / G_{1} \times G_{2}} \int_{G_{1} \times G_{2}}\left\langle\beta(x, y), L_{\eta}^{-1} f(\eta x) \otimes M_{\zeta}^{-1} g(\zeta y)\right\rangle d_{G_{1} \times G_{2}}(\eta, \zeta) \\
= & \int_{\mathscr{S} \times \mathscr{G} / G_{1} \times G_{2}}\left\langle\beta(x, y), \int_{G_{1}} L_{\eta}^{-1} f(\eta x) d_{G_{1}}(\eta) \otimes \int_{G_{2}} M_{\zeta}^{-1} g(\zeta y) d_{G_{2}}(\zeta)\right\rangle \\
= & \int_{\mathscr{S} \times \mathscr{G} / G_{1} \times G_{2}}\left\langle\beta(x, y), \pi_{L} f(x) \otimes v_{2}\right)(\bar{x}, \bar{y})
\end{aligned}
$$

Thus $B^{\prime}$ depends only on $\pi_{L} f$ and $\pi_{M} g$ and may be written 


$$
B^{\prime}(f, g)=B\left(\pi_{L} f, \pi_{M} g\right)
$$

where now, because $\pi_{L}$ and $\pi_{M}$ are homomorphisms of $L^{1}\left(\rho_{1} \mu, \boldsymbol{H}(L)\right)$ on $\boldsymbol{H}\left(V^{L}\right)$ and $L^{1}\left(\rho_{2} \mu, \boldsymbol{H}(M)\right)$ on $\boldsymbol{H}\left(V^{M}\right) B$ is a continuous bilinear form on $\boldsymbol{H}\left(V^{L}\right) \times \boldsymbol{H}\left(V^{M}\right)$. Because $\mathscr{G} \times \mathscr{G} / G_{1} \times G_{2}$ is canonically homeomorphic to $\mathscr{G} / G_{1} \times \mathscr{G} / G_{2}, B$ has the desired form (11). That $B$ is an intertwining form for $V^{L}$ and $V^{M}$ results from the equations:

$$
\begin{aligned}
B & \left(V_{s}^{L} \pi_{L} f, V_{s}^{M} \pi_{M} g\right)=B\left(\pi_{L} \tau_{s} f, \pi_{M} \tau_{s} g\right)=B^{\prime}\left(\tau_{s} f, \tau_{s} g\right) \\
& =\int_{\mathscr{S} \times g}\langle\beta(x, y), f(x s) \otimes g(y s)\rangle \frac{\rho_{1}(x s)}{\rho_{1}(x)} \frac{\rho_{2}(y s)}{\rho_{2}(y)} \rho_{1}(x) \rho_{2}(y) d_{\mathscr{G} \times \mathscr{G}}(x, y) \\
& =\int_{\mathscr{G} \times \mathscr{g}}\left\langle\beta\left(x s^{-1}, y s^{-1}\right), f(x) \otimes g(y)\right\rangle \rho_{1}(x) \rho_{2}(y) d_{\mathscr{G} \times \mathscr{G}}(x, y) \\
& =\int_{\mathscr{G} \times \mathscr{G}}\langle\beta(x, y), f(x) \otimes g(y)\rangle \rho_{1}(x) \rho_{2}(y) d_{\mathscr{G} \times \mathscr{G}}(x, y) \\
& =B^{\prime}(f, g)=B\left(\pi_{L} f, \pi_{M} g\right) .
\end{aligned}
$$

Thus we have shown that to each $\beta \in \boldsymbol{F}(L, M)$ there corresponds an intertwining form $B$ defined by (11), and to this $B$ corresponds exactly $\beta \in F(L, M)$, under the isometry described at the beginning of the proof. This completes the proof.

We now want to examine the functions $\beta \in \boldsymbol{F}(L, M)$. For each $x, y \in \mathscr{G} \times \mathscr{G}$, $\beta(x, y) \in(\boldsymbol{H}(L) \hat{\otimes} \boldsymbol{H}(M))^{\prime}$, that is, $\beta(x, y)$ is a continuous bilinear form on $\boldsymbol{H}(L) \times \boldsymbol{H}(M)$. We shall see that it is an intertwining form for certain representations related to $L$ and $M$.

For each $x, y \in \mathscr{G} \times \mathscr{G}$ set $G_{x, y}=x^{-1} G_{1} x \cap y^{-1} G_{2} y$. Let $L^{x}$ and $M^{y}$ be the representations $s \rightarrow L_{s}^{x}=L_{x s x^{-1}}$ and $s \rightarrow M_{s}^{y}=M_{y s y-1}$ of $G_{x, y}$. Let $B(L, M, x, y)$ denote the space of intertwining forms for the representations $L^{x}$ and $M^{y}$.

Lemma 3. If $\beta \in \boldsymbol{F}(L, M)$ then $\beta^{\prime}(x, y) \in \boldsymbol{B}(L, M, x, y)$, almost all $x, y \in \mathscr{G} \times \mathscr{G}$.

Proof. Suppose that for some fixed $x, y \in \mathscr{G} \times \mathscr{G}, \beta\left(\eta^{-1} x s, \zeta^{-1} y s\right)$ $={ }^{t}\left(L_{\eta} \times M_{\zeta}\right) \beta(x, y)$ for all $\eta, \zeta, s \in G_{1} \times G_{2} \times \mathscr{G}$. Then for all $\zeta \in G_{x, y}$

$$
{ }^{t}\left(L_{\xi}^{x} \otimes M_{\xi}^{y}\right) \beta(x, y)={ }^{t}\left(L_{x \xi x-1} \otimes M_{y \xi y}-1\right) \beta(x, y)=\beta\left(x \xi^{-1}, y \xi^{-1}\right)=\beta(x, y)
$$

and $\beta(x, y) \in \boldsymbol{B}(L, M, x, y)$.

Thus the functions $\beta$ corresponding to intertwining forms for $V^{L}$ and $V^{M}$ are measurable essentially bounded functions $\beta: \mathscr{G} \times \mathscr{G} \rightarrow(H(L) \hat{\otimes} \boldsymbol{H}(M))^{\prime}$ which in the first place have the property that $\beta(x, y) \in B(L, M, x, y)$, almost all $x, y \in \mathscr{G} \times \mathscr{G}$. In addition the condition (10) specifies that if $x_{1}, y_{1}$ and $x_{2}, y_{2}$ are two points in $\mathscr{G} \times \mathscr{G}$ such that $x_{1} y_{1}^{-1}$ and $x_{2} y_{2}^{-1}$ belong to the same $G_{1}: G_{2}$ double coset, the value of $\beta$ at $x_{1}, y_{1}$ completely determines its value at $x_{2}, y_{2}$. Let $\tilde{\mathscr{G}}$ be the 
diagonal of $\mathscr{G} \times \mathscr{G}$. Then $x_{1} y_{1}^{-1}$ and $x_{2} y_{2}^{-1}$ belong to the same $G_{1}: G_{2}$ double coset if and only if $x_{1}, y_{1}$ and $x_{2}, y_{2}$ belong to the same $G_{1} \times G_{2}: \tilde{G}$ double coset in $\mathscr{G} \times \mathscr{G}$. Thus we can say that once the value of $\beta$ is specified at a point $x, y$ it is uniquely determined on the double $\operatorname{coset}\left(G_{1} \times G_{2}\right)(x, y) \tilde{\mathscr{G}}$.

THEOREM 2. With the notation of Theorem 1 suppose that $\mathscr{G}$, as well as $\boldsymbol{H}(L)$ and $\boldsymbol{H}(M)$, is separable. Let $\tilde{G}$ be the diagonal of $\mathscr{G} \times \mathscr{G}$, let $\mathscr{D}$ be the set $G_{1} \times G_{2}: \tilde{G}$ double cosets and let $p: \mathscr{G} \times \mathscr{G} \rightarrow \mathscr{D}$ be the canonical map. Provide $\mathscr{D}$ with the quotient Borel structure and suppose that the image $\alpha$ in $\mathscr{D}$ of Haar measure on $\mathscr{G} \times \mathscr{G}$ is standard [5, p. 142], or what is equivalent, that $G_{1}$ and $G_{2}$ are regularly related $[4, \mathrm{p} .127]$. Then there exists a measurable cross section $\psi: \mathscr{D} \rightarrow \mathscr{G} \times \mathscr{G}$. For all $\beta \in \boldsymbol{F}(L, M), \beta^{0}=\beta \circ \psi \circ p$, identified to a function on $\mathscr{D}$, is in $L^{\infty}\left(\alpha,(H(L) \hat{\otimes} \boldsymbol{H}(M))^{\prime}\right)$. The map $\beta \rightarrow \beta^{0}$ is a linear isometry of $\boldsymbol{F}(L, M)$ on the subspace of all $\gamma \in L^{\infty}\left(\alpha,(\boldsymbol{H}(L) \hat{\otimes} \boldsymbol{H}(M))^{\prime}\right)$ such that $\gamma(p(x, y)) \in \boldsymbol{B}(L, M, \psi p(x, y))$, almost all $x, y \in \mathscr{G} \times \mathscr{G}$.

Proof. Because $\alpha$ is standard there exists a Borel subset $\mathscr{D}_{1}$ of $\mathscr{D}$ such that $\mathscr{D}_{1}$ is standard and $\alpha\left(\mathscr{C}_{1}\right)=0$. By a lemma of von Neumann, as formulated by Mackey [5, Theorem 6.3], there exists a Borel subset $\mathscr{D}_{2}$ of $\mathscr{D}$, such that $\alpha\left(\mathscr{C D}_{2}\right)=0$ and a Borel function $\psi: \mathscr{D}_{2} \rightarrow \mathscr{G} \times \mathscr{G}$ such that $p \psi(d)=d$, for all $d \in \mathscr{D}_{2}$, that is, $\psi$ is a Borel cross section of $\mathscr{D}_{2}$ in $p^{-1}\left(\mathscr{D}_{2}\right) \subset \mathscr{G} \times \mathscr{G}$. Moreover $S=\psi\left(\mathscr{D}_{2}\right)$ is a Borel subset of $\mathscr{G} \times \mathscr{G}$ by [5, Theorem 3.2], since $\mathscr{D}_{2}$ is a Borel subset of a standard Borel space and thus standard. Because $p^{-1}\left(\mathfrak{C D}_{2}\right)$ is of Haar measure 0 , we can extend $\psi$ to a cross section of $\mathscr{D}$ in $\mathscr{G} \times \mathscr{G}$ in any fashion, and be sure that the extended map, which we also call $\psi$, is measurable (with respect to the $\sigma$-field of Haar measurable sets).

For each $\beta \in F(L, M), \beta^{0}=\beta \circ \psi \circ p$, identified to a function on $\mathscr{D}$, is meassurable, because $\psi \circ p \mid p^{-1}\left(\mathscr{D}_{2}\right)$ is a Borel function and $\mathbf{C} p^{-1}\left(\mathscr{D}_{2}\right)=p^{-1}\left(\mathbf{C} \mathscr{D}_{2}\right)$ has Haar measure 0. For all $x, y \in \mathscr{G} \times \mathscr{G}, \psi p(x, y)=\eta x s, \zeta y s$, for some $\eta, \zeta, s \in G_{1} \times G_{2} \times \mathscr{G}$ and $\left\|\beta^{0}(x, y)\right\|=\|\beta(\eta x s, \zeta y s)\|=\left\|^{t}\left(L_{\eta}^{-1} \otimes M_{\zeta}^{-1}\right) \beta(x, y)\right\|$ $=\|\beta(x, y)\|$. Thus $\beta$ and $\beta^{0}$ have the same essential supremum. Thus

$$
\beta^{0} \in L^{\infty}\left(\alpha,(H(L) \hat{\otimes} H(M))^{\prime}\right)
$$

and $\beta \rightarrow \beta^{0}$ is an isometry. By Lemma $3, \beta^{0}(x, y)=\beta(\psi p(x, y)) \in \boldsymbol{B}(L, M, \psi p(x, y))$.

Choose $\gamma \in L^{\infty}\left(\alpha,(\boldsymbol{H}(L) \hat{\otimes} \boldsymbol{H}(M))^{\prime}\right)$ such that $\gamma(p(x, y)) \in \boldsymbol{B}(L, M, \psi p(x, y))$, almost all $x, y \in \mathscr{G} \times \mathscr{G}$. We define a function $\gamma^{\prime}$ on $\mathscr{G} \times \mathscr{G}$ by:

$$
\gamma^{\prime}(x, y)={ }^{t}\left(L_{\eta}^{-1} \otimes M_{\zeta}^{-1}\right) \gamma(p(x, y)) \text {, if } x, y=(\eta, \zeta) \psi p(x, y)(s, s), \eta, \zeta \in G_{1} \times G_{2} .
$$

We must verify that $\gamma^{\prime}$ is well defined almost everywhere. If

$$
\left(\eta_{1}, \zeta_{1}\right) \psi(p(x, y))\left(s_{1}, s_{1}\right)=(\eta, \zeta) \psi(p(x, y))(s, s)
$$

then, setting $\psi(p(x, y))=x_{1}, y_{1}$, 


$$
\xi=x_{1}^{-1} \eta^{-1} \eta_{1} x_{1}=y_{1}^{-1} \zeta^{-1} \zeta_{1} y_{1}=s_{1} s^{-1} \in G_{x_{1}, y_{1}} .
$$

But $\gamma^{\prime}\left(x_{1}, y_{1}\right) \in \boldsymbol{B}\left(L, M, x_{1}, y_{1}\right)$ so that

$$
{ }^{t}\left(L_{\xi}^{x_{1}} \otimes M_{\xi}^{y_{1}}\right) \gamma^{\prime}\left(x_{1}, y_{1}\right)=\gamma^{\prime}\left(x_{1}, y_{1}\right)
$$

and this says

$$
{ }^{t}\left(L_{\eta}^{-1} L_{\eta_{1}} \otimes M_{\zeta}^{-1} M_{\zeta_{1}}\right) \gamma^{\prime}\left(x_{1}, y_{1}\right)=\gamma^{\prime}\left(x_{1}, y_{1}\right),
$$

which in turn implies that

$$
{ }^{t}\left(L_{\eta_{1}}^{-1} \otimes M_{\zeta_{1}}^{-1}\right) \gamma^{\prime}\left(x_{1}, y_{1}\right)={ }^{t}\left(L_{\eta}^{-1} \otimes M_{\zeta}^{-1}\right) \gamma^{\prime}\left(x_{1}, y_{1}\right) .
$$

Thus $\gamma^{\prime}$ is well defined for all those $x, y$ such that $\gamma(p(x, y)) \in \boldsymbol{B}(L, M, \psi p(x, y))$, that is, almost everywhere. Clearly, $\gamma^{\prime}{ }^{0}=\gamma, \gamma^{\prime}$ has the properties(10), and $\gamma^{\prime}$ and $\gamma$ have the same essential supremum. The only fact remaining to verify is that $\gamma^{\prime}$ is measurable.

Consider the set $S=\psi\left(\mathscr{D}_{2}\right): S$ is a Borel set which intersects each double coset contained in $p^{-1}\left(\mathscr{D}_{2}\right)$ in exactly one point. Suppose we have found a Borel set $N$ of measure 0 and a Borel function

$$
z \rightarrow \eta(z), \zeta(z), s(z): p^{-1}\left(\mathscr{D}_{2}\right) \cap \mathrm{C} N \rightarrow G_{1} \times G_{2} \times \mathscr{G}
$$

such that for all $z \in p^{-1}\left(\mathscr{D}_{2}\right) \cap \mathrm{CN},(\eta(z), \zeta(z)) z(s(z), s(z)) \in S$. Then

$$
\gamma^{\prime}(z)={ }^{t}\left(L_{\eta(z)}^{-1} \otimes M_{\zeta(z)}^{-1}\right) \gamma(p(z)), \quad z \in p^{-1}\left(\mathscr{D}_{2}\right) \cap \mathbf{G N},
$$

and $z \rightarrow \gamma^{\prime}(z)$ is a measurable function on $p^{-1}\left(\mathscr{D}_{2}\right) \cap \mathrm{GN}$. Again, because C $p^{-1}\left(\mathscr{D}_{2}\right)$ has Haar measure $0, \gamma^{\prime}$ will be a measurable function on $\mathscr{G} \times \mathscr{G}$.

The map $\eta, \zeta, s, z \rightarrow(\eta, \zeta) z\left(s^{-1}, s^{-1}\right): G_{1} \times G_{2} \times \mathscr{G} \times \mathscr{G} \times \mathscr{G} \rightarrow \mathscr{G} \times \mathscr{G}$ is a continuous function and because $p^{-1}\left(\mathscr{D}_{2}\right)$ is a union of double cosets, the restriction $h$ of this map to $G_{1} \times G_{2} \times \mathscr{G} \times p^{-1}\left(\mathscr{D}_{2}\right)$ is a Borel function $G_{1} \times G_{2} \times \mathscr{G} \times p^{-1}\left(\mathscr{D}_{2}\right) \rightarrow p^{-1}\left(\mathscr{D}_{2}\right)$. Moreover, the map which sends each $\eta, \zeta, s \in G_{1} \times G_{2} \times \mathscr{G}$ into the function $z \rightarrow(\eta, \zeta) z\left(s^{-1}, s^{-1}\right)$ is an isomorphism of $G_{1} \times G_{2} \times \mathscr{G}$ into the group of Borel isomorphisms of $p^{-1}\left(\mathscr{D}_{2}\right)$. Thus $G_{1} \times G_{2} \times \mathscr{G}$, $p^{-1}\left(\mathscr{D}_{2}\right), h$ is a Borel transformation group. Because $p^{-1}\left(\mathscr{D}_{2}\right)$ is a Borel subset of a standard Borel space, it itself is standard [5, Theorem 3.2, Corollary 1]. The existence of the required function $z \rightarrow \eta(z), \zeta(z), s(z)$ is a consequence of the following lemma which in turn is a consequence of the lemma of von Neumann.

LEMMA 4. Let $\mathscr{G}, X, h$ be a Borel transformation group, let $S$ be a Borel cross section of the orbits in $X$ and let $\mu$ be a Borel measure on $X$. Suppose $\mathscr{G}$ and $X$ are standard Borel spaces. Then there exists a Borel set $N \subset X$ such that $\mu(N)=0$, and a Borel function $x \rightarrow g(x): C N \rightarrow \mathscr{G}$ such that $g(x) \cdot x \in S$ for all $x \notin N$. 
Proof. Consider the Borel set $h^{-1}(S) \subset X \times \mathscr{G}$. Because $S$ is a cross section of the orbits there exists for each $x \in X, g \in \mathscr{G}$ such that $g \cdot x \in S$, that is for each $x \in X$ there exists $g \in \mathscr{G}$ such that $x, g \in h^{-1}(S)$. By [5, Theorem 6.3] there exists a Borel set $N \subset X$ such that $\mu(N)=0$ and a Borel function $x \rightarrow g(x): G N \rightarrow \mathscr{G}$ such that $x, g(x) \in h^{-1}(S)$ for all $x \in \mathrm{CN}$. But $x, g(x) \in h^{-1}(S)$ if and only if $g(x) \cdot x \in S$, and $x \rightarrow g(x)$ is the desired function.

Suppose that $G_{2}=\mathscr{G}$. Then there is only one $G_{1} \times \mathscr{G}: \tilde{\mathscr{G}}$ double coset, namely the one containing e,e. $L^{e}=L$ and $M^{e}=M \mid G$. Moreover $L^{\infty}\left(\alpha,(\boldsymbol{H}(L) \hat{\otimes} \boldsymbol{H}(M))^{\prime}\right)$ $=(\boldsymbol{H}(L) \hat{\otimes} \boldsymbol{H}(M))^{\prime}$. Thus as an immediate corollary of Theorems 1 and 2 we have the following version of the Frobenius Reciprocity Theorem, which is a slight improvement of a version due to C. Moore [7].

THEOREM 3. Let $G$ be a closed subgroup of the separable locally compact group $\mathscr{G}$, let $L$ be a representation of $G$ and $M$ a representation of $\mathscr{G}$, both on separable Banach spaces. Then $\boldsymbol{B}\left(V^{L}, M\right)$ is canonically linearly isometric to $B(L, M \mid G)$. If $M$ is a representation on a separable reflexive Banach space, $\operatorname{Hom}_{\mathscr{g}}\left(V^{L}, M\right)$ is linearly isometric to $\operatorname{Hom}_{G}(L, M \mid G)$.

The last statement follows from the observations that $B\left(V^{L}, M\right)=\operatorname{Hom}_{g}\left(V^{L}, \check{M}\right)$, and that if $\boldsymbol{H}(M)$ is reflexive, $(\breve{M})^{2}=M$.

COROLlARY (OSIMA RECIPROCITY THEOREM). With the notation and hypotheses of Theorem 2, $\boldsymbol{B}\left(V^{L} \mid G_{2}, M\right)$ is linearly isometric to $\boldsymbol{B}\left(L, V^{M} \mid G_{1}\right)$.

In fact, by two applications of Theorem $3, \boldsymbol{B}\left(V^{L} \mid G_{2}, M\right)$ is linearly isometric to $\boldsymbol{B}\left(V^{L}, V^{M}\right)$, which in turn is linearly isometric to $B\left(L, V^{M} \mid G_{1}\right)$.

4. Intertwining numbers. In this section we shall determine the intertwining number $i\left(V^{L}, V^{M}\right)=\operatorname{dim} B\left(V^{L}, V^{M}\right)$ which is, according to Theorem 2, the dimension of a certain subspace of $L^{\infty}\left(\alpha,(H(L) \hat{\otimes} H(M))^{\prime}\right)$. For this the following notion will be convenient.

Let $\Lambda$ and $X$ be topological spaces. We shall say that a family $\left(S_{\lambda}\right)_{\lambda \in \Lambda}$ of subsets of $X$ is closed if

$$
\operatorname{gr}\left(\left(S_{\lambda}\right)\right)=\left\{\lambda, x: x \in S_{\lambda}\right\}
$$

is closed in $\Lambda \times X$.

LEMma 5. Let $\Lambda, \Gamma, X$ be topological spaces and let $\left(S_{\lambda}\right)_{\lambda \in \Lambda}$ and $\left(T_{\gamma}\right)_{\gamma \in \Gamma}$ be two closed families of subsets of $X$. Then $\left(S_{\lambda} \cap T_{\gamma}\right)_{\lambda, \gamma \in \Lambda \times \Gamma}$ is a closed family.

Proof. The set $\operatorname{gr}\left(\left(S_{\lambda}\right)\right) \times \operatorname{gr}\left(\left(T_{\gamma}\right)\right)$ is a closed subset of $\Lambda \times X \times \Gamma \times X$ which is canonically homeomorphic to $\Lambda \times \Gamma \times X \times X$ under the map $\lambda, x, \gamma, y \rightarrow \lambda, \gamma, x, y$. Under this map $\operatorname{gr}\left(\left(S_{\lambda}\right)\right) \times \operatorname{gr}\left(\left(T_{\gamma}\right)\right)$ corresponds to the set of all $\lambda, \gamma, x, y \in \Lambda \times \Gamma \times X \times X$ such that $x, y \in S_{\lambda} \times T_{\gamma}$ and this is exactly the set $\operatorname{gr}\left(\left(S_{\lambda} \times T_{\gamma}\right)\right)$ which is accordingly closed. Let $\tilde{X}$ be the diagonal of $X \times X$. 
Then $\operatorname{gr}\left(\left(S_{\lambda} \times T_{\gamma}\right)\right) \cap \Lambda \times \Gamma \times \tilde{X}$ is a closed subset of $\Lambda \times \Gamma \times X$. The map $\theta: \lambda, \gamma, x, x \rightarrow \lambda, \gamma, x$ is a homeomorphism of $\Lambda \times \Gamma \times \tilde{X}$ with $\Lambda \times \Gamma \times X$. Now $\lambda, \gamma, x \in \theta\left(\operatorname{gr}\left(\left(S_{\lambda} \times T_{\gamma}\right)\right) \cap \Lambda \times \Gamma \times \widetilde{X}\right)$ if and only if $\lambda, \gamma, x, x \in \operatorname{gr}\left(\left(S_{\lambda} \times T_{\gamma}\right)\right)$ $\cap \Lambda \times \Gamma \times \tilde{X}$, that is, if and only if $x, x \in S_{\lambda} \times T_{\gamma}$, and this happens if and only if $x \in S_{\lambda} \cap T_{\gamma}$. Thus $\theta\left(\operatorname{gr}\left(\left(S_{\lambda} \times T_{\gamma}\right)\right) \cap \Lambda \times \Gamma \times \tilde{X}\right)=\operatorname{gr}\left(\left(S_{\lambda} \cap T_{\gamma}\right)\right)$ which is thus closed in $\Lambda \times \Gamma \times X$.

LEMMA 6. Let $\mathscr{G}$ be a topological group and let $G$ be a closed subgroup. The family $\left(x^{-1} G x\right)_{x \in \mathscr{G}}$ is closed.

Proof. The set of all $x, y \in \mathscr{G} \times \mathscr{G}$ such that $y \in x^{-1} G x$ is the same as the set of all $x, y$ such that $x y x^{-1} \in G$. Because the map $x, y \rightarrow x y x^{-1}: \mathscr{G} \times \mathscr{G} \rightarrow \mathscr{G}$ is continuous, the set of all $x, y$ such that $x y x^{-1} \in G$ is closed in $\mathscr{G} \times \mathscr{G}$. But this set is exactly $g r\left(\left(x^{-1} G x\right)\right)$.

COROllary. Let $G_{1}$ and $G_{2}$ be closed subgroups of $\mathscr{G}$ and set $G_{x, y}=x^{-1} G_{1} x \cap y^{-1} G_{2} y$. Then $\left(G_{x, y}\right)_{x, y \in \mathscr{G} \times \mathscr{G}}$ is a closed family.

This is an immediate consequence of the preceding two lemmas.

LEMma 7. With the notation of the corollary, for each $s \in \mathscr{G}$ let $N_{s}$ be the set of all $x, y \in \mathscr{G} \times \mathscr{G}$ such that $s \in G_{x, y}$. Then $N_{s}$ is closed.

Proof. $x, y \in N_{s}$ if and only if $s \in G_{x, y}$, that is, if and only if $x, y, s \in \operatorname{gr}\left(\left(G_{x, y}\right)\right)$. Because $\operatorname{gr}\left(\left(G_{x, y}\right)\right)$ is closed in $\mathscr{G} \times \mathscr{G} \times \mathscr{G}, N_{s}$ is closed.

LEMMA 8. Let $E$ be a locally convex space, let $A$ be a weakly total subset of $E^{\prime}$ (total for $\sigma\left(E, E^{\prime}\right)$ ) and denote by $\mathscr{L}_{A}(E)$ the space of all continuous linear operators on $E$ provided with the weakest topology for which all the maps $T \rightarrow\left\langle x^{\prime}, T x\right\rangle, x^{\prime} \in A, x \in E$, are continuous. Let $f: \Lambda \rightarrow \mathscr{L}_{A}(E)$ be a continuous function, and set $K_{\lambda}=\operatorname{ker} f(\lambda)$. Then $\left(K_{\lambda}\right)_{\lambda \in \Lambda}$ is a closed family of subspaces of $E$.

Proof. For each $x^{\prime} \in A$ set $\phi_{x^{\prime}}(\lambda, x)=\left\langle x^{\prime}, f(\lambda) x\right\rangle$. Then $x \in \operatorname{ker} f(\lambda)$ if and only if $\phi_{x^{\prime}}(\lambda, x)=0$ for all $x^{\prime} \in A$. Thus $\operatorname{gr}\left(\left(K_{\lambda}\right)\right)=\bigcap_{x^{\prime} \in A} \phi_{x^{\prime}}^{-1}(0)$ which is closed, since each $\phi_{x^{\prime}}$ is continuous.

Let $G_{1}$ and $G_{2}$ be closed subgroups of the locally compact group $\mathscr{G}$ and let $L$ and $M$ be representations of $G_{1}$ and $G_{2}$ respectively. We shall apply the previous results to show that $(B(L, M, x, y))_{x, y \in \mathscr{G} \times \mathscr{G}}$ is a closed family of subspaces of $(\boldsymbol{H}(L) \hat{\otimes} \boldsymbol{H}(M))_{s}^{\prime}$, where $(\boldsymbol{H}(L) \hat{\otimes} \boldsymbol{H}(M))_{s}^{\prime}$ denotes the space $(\boldsymbol{H}(L) \hat{\otimes} \boldsymbol{H}(M))^{\prime}$ equipped with the topology $\sigma\left((\boldsymbol{H}(L) \hat{\otimes} \boldsymbol{H}(M))^{\prime}, \boldsymbol{H}(L) \hat{\otimes} \boldsymbol{H}(M)\right)$.

Note that $B \in \boldsymbol{B}(L, M, x, y)$ if and only if

$$
{ }^{t}\left(L_{s}^{x} \otimes M_{s}^{y}\right) B=B, \quad \text { all } s \in G_{x, y},
$$

that is, if and only if

$$
B \in \operatorname{ker}\left({ }^{t}\left(L_{s}^{x} \otimes M_{s}^{y}\right)-I\right), \quad \text { all } s \in G_{x, y},
$$


where $I$ is the identity operator. Because strongly continuous linear operators on $(\boldsymbol{H}(L) \hat{\otimes} \boldsymbol{H}(M))^{\prime}$ are also continuous linear operators on $(\boldsymbol{H}(L) \hat{\otimes} \boldsymbol{H}(M))_{s}^{\prime}$, ${ }^{t}\left(L_{s}^{x} \otimes M_{s}^{y}\right)-I \in \mathscr{L}_{A}\left((\boldsymbol{H}(L) \otimes \boldsymbol{H}(M))_{s}^{\prime}\right)$, where $A=\boldsymbol{H}(L) \otimes \boldsymbol{H}(M)$. For each $s \in \mathscr{G}$ let $R_{s}$ be the function defined on $N_{s}$ (Lemma 7) with values in $\mathscr{L}_{A}\left((\boldsymbol{H}(L) \hat{\otimes} \boldsymbol{H}(M))_{s}^{\prime}\right)$ defined by:

$$
R_{s}^{x, y}={ }^{t}\left(L_{s}^{x} \otimes M_{s}^{y}\right)-I .
$$

For each $s \in \mathscr{G}, x \rightarrow x s x^{-1}$ and $y \rightarrow y s y^{-1}$ are continuous. Thus $x, y \rightarrow^{t}\left(L_{s}^{x} \otimes M_{s}^{y}\right)$, restricted to $N_{s}$, is a continuous function $N_{s} \rightarrow \mathscr{L}_{A}\left((\boldsymbol{H}(L) \hat{\otimes} \boldsymbol{H}(M))_{s}^{\prime}\right)$, and thus $R_{s}$ is continuous.

LEMMA 9. $(\boldsymbol{B}(L, M, x, y))_{x, y \in \mathscr{G} \times \mathscr{G}}$ is a closed family of subspaces of $(\boldsymbol{H}(L) \hat{\otimes} \boldsymbol{H}(M))_{s}^{\prime}$.

Proof. Because $R_{s}$ is continuous, it follows from Lemma 8 that $\left(\operatorname{ker} R_{s}^{x, y}\right)_{x, y \in N_{s}}$ is a closed family of subspaces of $(H(L) \hat{\otimes} H(M))_{s}^{\prime}$. Thus $\operatorname{gr}\left(\left(\operatorname{ker} R_{s}^{x, y}\right)\right)$ is a closed subset of $N_{s} \times(\boldsymbol{H}(L) \hat{\otimes} \boldsymbol{H}(M))_{s}^{\prime} \subset \mathscr{G} \times \mathscr{G} \times(\boldsymbol{H}(L) \hat{\otimes} \boldsymbol{H}(M))_{s}^{\prime}$, for each $s \in \mathscr{G}$. Hence

$$
K=\bigcap_{s \in \mathscr{G}} g r\left(\left(\operatorname{ker} R_{s}^{x, y}\right)\right)
$$

is closed in $\mathscr{G} \times \mathscr{G} \times(\boldsymbol{H}(L) \hat{\otimes} \boldsymbol{H}(M))_{s}^{\prime}$. But $x, y, B \in K$ if and only if $B \in \operatorname{gr}\left(\left(\operatorname{ker} R_{s}^{x, y}\right)\right)$ for all $s \in \mathscr{G}$, that is, if and only if ${ }^{t}\left(L_{s}^{x} \otimes M_{s}^{y}\right) B=B$ for all $x, y \in N_{s}$ and $s \in \mathscr{G}$, and this happens if and only if ${ }^{t}\left(L_{s}^{x} \otimes M_{s}^{y}\right) B=B$ for all $s \in G_{x, y}$ and all $x, y \in \mathscr{G} \times \mathscr{G}$, i.e., if and only if $B \in \boldsymbol{B}(L, M, x, y)$, for all $x, y \in \mathscr{G} \times \mathscr{G}$. Hence $K=\operatorname{gr}(\boldsymbol{B}(L, M, x, y))$ which is accordingly closed, and this proves the lemma.

Suppose that $G_{1}$ and $G_{2}$ are regularly related. Let $\mathscr{D}$ be the space of $G_{1} \times G_{2}: \tilde{\mathscr{G}}$ double cosets and let $\alpha$ be the image in $\mathscr{D}$ of Haar measure. Then there exists a Borel subset $\mathscr{D}_{2}$ of $\mathscr{D}$ such that $\mathscr{D}_{2}$ is a standard Borel space and $\alpha\left(\mathscr{C}_{2}\right)=0$, and a measurable cross section $\psi: \mathscr{D} \rightarrow \mathscr{G} \times \mathscr{G}$ such that $\psi \mathscr{D}_{2}$ is a Borel subset of $\mathscr{G} \times \mathscr{G}$. In $\mathscr{D}_{2} \times(\boldsymbol{H}(L) \hat{\otimes} \boldsymbol{H}(M))_{s}^{\prime}$ the set of all $a, B$ such that $B \in \boldsymbol{B}(L, M, \psi a)$ is Borel isomorphic to $\operatorname{gr}(\boldsymbol{B}(L, M, x, y)) \cap \psi \mathscr{D}_{2} \times(\boldsymbol{H}(L) \hat{\otimes} \boldsymbol{H}(M))_{s}^{\prime}$. This last set is a Borel set so that the set of all $a, B \in \mathscr{D}_{2} \times(\boldsymbol{H}(L) \hat{\otimes} \boldsymbol{H}(M))_{s}^{\prime}$ such that $B \in \boldsymbol{B}(L, M, \psi a)$ is a Borel subset of $\mathscr{D}_{2} \times(\boldsymbol{H}(L) \hat{\otimes} \boldsymbol{H}(M))_{s}^{\prime}$. The subspace of $L^{\infty}\left(\alpha,(\boldsymbol{H}(L) \hat{\otimes} \boldsymbol{H}(M))_{s}^{\prime}\right)$ of all $f$ such that $f(a) \in \boldsymbol{B}(L, M, \psi a), \alpha$ almost all $a \in \mathscr{D}$, is the same as the subspace of all those $f$ such that $f(a) \in \boldsymbol{B}(L, M, \psi a), \alpha$ almost all $a \in \mathscr{D}_{2}$. Thus we are in the following situation.

Let $\alpha$ be a Borel measure on the Borel space $\Lambda$, let $E$ be a Banach space and let $\left(E_{\lambda}^{\prime}\right)_{\lambda \in \Lambda}$ be a Borel family of subspaces of $E_{s}^{\prime}$ in the sense that $\operatorname{gr}\left(\left(E_{\lambda}^{\prime}\right)\right)$ is a Borel subset of $\Lambda \times E_{s}^{\prime}$, where $E_{s}^{\prime}$ denotes $E^{\prime}$ equipped with $\sigma\left(E^{\prime}, E\right)$. Denote by $L^{\infty}\left(\alpha,\left(E_{\lambda}^{\prime}\right)\right)$ the subspace of $L^{\infty}\left(\alpha, E_{s}^{\prime}\right)$ consisting of all $f$ such that $f(\lambda) \in E_{\lambda}^{\prime}, \alpha$ almost all $\lambda$. The problem is to compute $\operatorname{dim} L^{\infty}\left(\alpha,\left(E_{\lambda}^{\prime}\right)\right)$.

Lemma 10. Let $\alpha$ be a standard Borel measure on the Borel space $\Lambda$ and let $E$ be a separable Banach space. Let $\left(E_{\lambda}^{\prime}\right)_{\lambda \in \Lambda}$ be a Borel family of closed subspaces 
of $E_{s}^{\prime}$. Write $\alpha=\alpha_{1}+\alpha_{2}$ where $\alpha_{1}$ is a purely atomic Borel measure and $\alpha_{2}$ is a nonatomic Borel measure. If $E_{\lambda}^{\prime} \neq 0$ on a measurable set of positive $\alpha_{2}$ measure, $\operatorname{dim} L^{\infty}\left(\alpha,\left(E_{\lambda}^{\prime}\right)\right)=\infty$. If $E_{\lambda}^{\prime}=0, \alpha_{2}$ almost everywhere,

$$
\operatorname{dim} L^{\infty}\left(\alpha,\left(E_{\lambda}^{\prime}\right)\right)=\int \operatorname{dim} E_{\lambda}^{\prime} d \alpha_{1}(\lambda)
$$

Proof. Suppose $E_{\lambda}^{\prime} \neq 0$ on the measurable set $\Lambda_{1}$ of positive $\alpha_{2}$ measure. Let $P$ be the unit ball in $E^{\prime}$. Then $2 P$ is a compact metrizable subset of $E_{s}^{\prime}$, and $\Lambda_{1} \times 2 P \cap \operatorname{gr}\left(\left(E_{\lambda}^{\prime}\right)\right)$ is a Borel subset of $\Lambda_{1} \times 2 P$. Thus

$$
T=\left(\Lambda_{1} \times(2 P \cap \mathbf{C P})\right) \cap \operatorname{gr}\left(\left(E_{\lambda}^{\prime}\right)\right)
$$

is also a Borel subset of $\Lambda_{1} \times 2 P$, and for each $\lambda \in \Lambda$, there exists $x \in E_{\lambda}^{\prime}$ such that $\lambda, x \in T$. Because $2 P$ is a compact metrizable space, it is a standard Borel space and by the lemma of von Neumann [5, Theorem 6.3] there exists a Borel subset $\Lambda_{2} \subset \Lambda_{1}$ such that $\alpha\left(\Lambda_{1} \cap \mathrm{C} \Lambda_{2}\right)=0$ and a Borel function $g: \Lambda_{2} \rightarrow E^{\prime}$ such that $\lambda, g(\lambda) \in T$ for all $\lambda \in \Lambda_{2}$. In particular, $1<\|g(\lambda)\| \leqq 2$, for all $\lambda \in \Lambda_{2}$. Extend $g$ to all of $\Lambda$ by setting $g(\lambda)=0$ for $\lambda \notin \Lambda_{2}$. Then $g \in L^{\infty}\left(\alpha,\left(E_{\lambda}^{\prime}\right)\right)$. Because $\alpha_{2}$ is $\sigma$-finite and nonatomic we can write $\Lambda_{2}$ as a countable disjoint union of Borel sets $\Lambda_{2, j}, j=1,2, \cdots$, each of positive $\alpha_{2}$ measure. Set $g_{j}(x)=g(x), x \in \Lambda_{2, j}$, $g_{j}(x)=0, x \notin \Lambda_{2, j}$. Then each $g_{j} \in L^{\infty}\left(\alpha,\left(E_{\lambda}^{\prime}\right)\right)$ and the $g_{j}$, or rather their classes, are linearly independent, so that $\operatorname{dim} L^{\infty}\left(\alpha,\left(E_{\lambda}\right)\right)=\infty$.

Suppose that $E_{\lambda}^{\prime}=0, \alpha_{2}$ almost everywhere. We shall see, as was pointed out to me by $\mathbf{J}$. Auslander, that the only atoms for $\alpha$ are points. Because $\alpha$ is standard there exists a Borel subset $\Lambda_{0}$ of $\Lambda$ such that $\alpha\left(C \Lambda_{0}\right)=0$ and $\Lambda_{0}$ is a standard Borel space, hence Borel isomorphic to the unit interval $I$ or to an at most countably infinite set $J$. If $\Lambda_{0}$ is isomorphic to $J$ then clearly the only atoms are points. Suppose that $\Lambda_{0}$ is Borel isomorphic to $I$.

If $A$ is a Borel subset of $I$ of positive $\alpha$ measure which is an atom, and we write $I=I_{1} \cup I_{2}$ where $I_{1}=[0,1 / 2), I_{2}=[1 / 2,1]$, then exactly one of $A_{1}=A \cap I_{1}$, or $A_{2}=A \cap I_{2}$ has positive $\alpha$ measure and the other has 0 measure, say $\alpha\left(A_{1}\right)=\alpha(A)>0$. If we repeat this bisection indefinitely we see that if $A$ is an atom, then for each $n>0, A$ contains a Borel subset of diameter $<2^{-n}$ such that $\alpha(A)=\alpha\left(A_{n}\right)$. Thus $A$ must contain a point $\lambda_{0}$ such that $\alpha\left(\left\{\lambda_{0}\right\}\right)=\alpha(A)$, and the only atoms are those which differ from points by null sets. But now $L^{\infty}\left(\alpha,\left(E_{\lambda}^{\prime}\right)\right)$ is clearly the $L^{\infty}$ sum of those spaces $E_{\lambda_{i}}^{\prime}$ such that $\alpha\left(\left\{\lambda_{i}\right\}\right)>0$, and the dimension of this space is $\sum_{i} \operatorname{dim} E_{\lambda_{i}}^{\prime}$. Since $\operatorname{dim} E_{\lambda}^{\prime}$ is measurable for $\alpha_{1}$ we can write this sum as an integral $\int \operatorname{dim} E_{\lambda}^{\prime} d \alpha_{1}(\lambda)$, and this proves the second part of the lemma.

LEMMA 11. For all $\eta, \zeta, s \in G_{1} \times G_{2} \times \mathscr{G}$,

$$
\boldsymbol{B}(L, M: \eta x s, \zeta y s)={ }^{t}\left(L_{\eta}^{-1} \otimes M_{\zeta}^{-1}\right) \boldsymbol{B}(L, M, x, y) .
$$

In particular, $\operatorname{dim} B(L, M, x, y)$ depends only on the $G_{1} \times G_{2}: \tilde{\mathscr{G}}$ double coset $a$ to which $x, y$ belongs, and may be written $i(L, M, a)$. 
Proof. Note that for each $s \in \mathscr{G}, G_{x s, y s}=s^{-1} G_{x, y}$. Thus, if $B \in B(L, M, x, y)$, $s \in \mathscr{G}, \xi \in G_{x s, y s}$ then $s s^{-1} \in G_{x, y}$ and

$$
{ }^{t}\left(L_{\xi}^{x s} \otimes M_{\xi}^{y s}\right) B={ }^{t}\left(L_{s \xi_{s}-1}^{x} \otimes M_{s \xi s-1}^{y}\right) B=B,
$$

and $B \in B(L, M, x s, y s)$. The same argument with $x, y$ replaced by $x s, y s$ and $s$ by $s^{-1}$ shows that $B(L, M, x, y)=B(L, M, x s, y s)$. Choose $\eta, \zeta \in G_{1} \times G_{2}$. Then $G_{\eta x, \zeta y}=G_{x, y}$ and for all $\xi \in G_{x, y}$,

$$
\begin{aligned}
{ }^{t}\left(L_{\xi}^{\eta x} \otimes M_{\xi}^{\zeta y}\right){ }^{t}\left(L_{\eta}^{-1} \otimes M_{\zeta}^{-1}\right) B & ={ }^{t}\left(L_{x \xi x}-1 \eta^{-1} \otimes M_{y \xi y-1 \zeta-1}\right) B \\
& ={ }^{t}\left(L_{\eta}^{-1} \otimes M_{\zeta}^{-1}\right)^{t}\left(L_{\xi}^{x} \otimes M_{\xi}^{y}\right) B={ }^{t}\left(L_{\eta}^{-1} \otimes M_{\zeta}^{-1}\right) B .
\end{aligned}
$$

Hence, for all $B \in \boldsymbol{B}(L, M, x, y),{ }^{t}\left(L_{\eta}^{-1} \otimes M_{\zeta}^{-1}\right) B \in B(L, M, \eta x, \zeta y)$. Repeating the same argument with $x, y$ repiaced by $\eta^{-1} x, \zeta^{-1} y$ shows that $B(L, M, \eta x, \zeta y)$ $={ }^{t}\left(L_{\eta}^{-1} \otimes M_{\zeta}^{-1}\right) B(L, M, x, y)$. These two results prove the first part of the lemma. Because $^{t}\left(L_{\eta}^{-1} \otimes M_{\zeta}^{-1}\right)$ is an isometry,

$$
\operatorname{dim} \boldsymbol{B}(L, M, \eta x s, \zeta y s)=\operatorname{dim}\left({ }^{t}\left(L_{\eta}^{-1} \otimes M_{\zeta}^{-1}\right) \boldsymbol{B}(L, M, x, y)\right)=\operatorname{dim} \boldsymbol{B}(L, M, x, y),
$$

and the dimension only depends on the $G_{1} \times G_{2}: \tilde{\mathscr{G}}$ double coset to which $x, y$ belongs.

Taking into account the last two lemmas and the remarks preceding Lemma 10 , we can state the following intertwining number theorem.

THEOREM 4. With the notation and hypotheses of Theorem 2, write $\alpha=\alpha_{1}+\alpha_{2}$ where $\alpha_{1}$ is a purely atomic Borel measure and $\alpha_{2}$ is nonatomic measure. If $i(L, M, a)>0$ on a set of positive $\alpha_{2}$ measure, $i\left(V^{L}, V^{M}\right)=\infty$. If $i(L, M, a)=0$, $\alpha_{2}$ almost everywhere, then

$$
i\left(V^{L}, V^{M}\right)=\int i(L, M, a) d \alpha_{1}(a) .
$$

5. Square summable induced representations. Let $G$ be a closed subgroup of the locally compact group $\mathscr{G}$ and let $L$ be a representation of $G$. Let $\boldsymbol{H}\left(U^{L}\right)$ be the set of all (classes of) measurable functions $f: \mathscr{G} \rightarrow \boldsymbol{H}(L)$ such that

(a) $f(\xi x)=L_{\xi} f(x), \xi, x \in G \times \mathscr{G}$,

(b) $\|f\|=\left(\int_{\mathscr{G} / G}\|f(x)\|^{2} d v(\bar{x})\right)^{1 / 2}<\infty$,

where again the meaning of the integral in (b) depends upon the fact that by (a), $\|f(x)\|^{2}$ depends only on $\bar{x}$. With the indicated norm, $H\left(U^{L}\right)$ is a normed space, and a Banach space if there exists a Borel cross section of $\mathscr{G} / G$ in $\mathscr{G}$. If $\boldsymbol{H}(L)$ is a Hilbert space, $\boldsymbol{H}\left(U^{L}\right)$ is at least a pre-Hilbert space.

For each $s \in \mathscr{G}$ set

$$
U_{s}^{L} f(x)=(\rho(x s) / \rho(x))^{1 / 2} f(x s) .
$$

Then $U_{s}^{L}$ is an isometry of $\boldsymbol{H}\left(U^{L}\right)$, and if $\boldsymbol{H}\left(U^{L}\right)$ is complete, $s \rightarrow U_{s}^{L}$ is a rep- 
resentation of $\mathscr{G}$, called the square summable represents :ion of $\mathscr{G}$ induced from $L$. If $L$ is a unitary representation, so is $U^{L}$ (assuming of course that $\boldsymbol{H}\left(U^{L}\right)$ is complete) and $U^{L}$ is just the unitary induced representation defined by Mackey [4].

Suppose there exists an invariant measure on $\mathscr{G} / G$ so that we can take $\rho=1$. Then $\boldsymbol{H}\left(U^{L}\right) \cap \boldsymbol{H}\left(V^{L}\right)$ is dense in both the containing spaces and $V_{s}^{L}\left|\boldsymbol{H}\left(U^{L}\right) \cap \boldsymbol{H}\left(V^{L}\right)=U_{s}^{L}\right| \boldsymbol{H}\left(U^{L}\right) \cap \boldsymbol{H}\left(V^{L}\right)$. If, further, there exists a finite invariant measure on $\mathscr{G} / G, H\left(U^{L}\right) \subset \boldsymbol{H}\left(V^{L}\right)$ and the inclusion map is continuous, of norm $\leqq 1$.

THEOREM 5. Let $G_{i}$ be a closed $\sigma$-compact subgroup of the locally compact group $\mathscr{G}$ such that there exists a Borel cross section of $\mathscr{G} / G_{i}$ in $\mathscr{G}$ and such that there exists a finite invariant measure on $\mathscr{G} / G_{i}, i=1,2$. Let $L$ and $M$ be representations of $G_{1}$ and $G_{2}$ respectively. The map $B \rightarrow B \mid \boldsymbol{H}\left(U^{L}\right) \times \boldsymbol{H}\left(U^{M}\right)$ is a continuous linear injection $\left(\boldsymbol{H}\left(V^{L}\right) \hat{\otimes} \boldsymbol{H}\left(V^{M}\right)\right)^{\prime} \rightarrow\left(\boldsymbol{H}\left(U^{L}\right) \hat{\otimes} \boldsymbol{H}\left(U^{M}\right)\right)^{\prime}$ and under this map $\boldsymbol{B}\left(V^{L}, V^{M}\right)$ is carried onto a subspace of $\boldsymbol{B}\left(U^{L}, U^{M}\right)$. Thus $i\left(V^{L}, V^{M}\right) \leqq i\left(U^{L}, U^{M}\right)$.

Proof. All that need be noted is that, under the hypotheses stated, $U_{s}^{L}=V_{s}^{L} \mid \boldsymbol{H}\left(U^{L}\right)$ and $U_{s}^{M}=V_{s}^{M} \mid \boldsymbol{H}\left(U^{M}\right)$ for all $s \in \mathscr{G}$.

Suppose that $L$ and $M$ are unitary representations. With the canonical conjugatelinear identification of $\boldsymbol{H}(L)$ with $\boldsymbol{H}(L)^{\prime}, \breve{L}$ is the conjugate representation $\bar{L}$ of $L$. Similarly, $\left(U^{L}\right)^{\smile}$ is equivalent to $\left(U^{L}\right)^{-}$which in turn is equivalent to $U^{\bar{L}}[4$, Theorem 5.1] and $\left(U^{M}\right)^{\smile}$ is equivalent to $U^{\bar{M}}$. $\operatorname{Hom}_{\mathscr{g}}\left(U^{L}, U^{M}\right)$ is isomorphic to $\boldsymbol{B}\left(U^{L},\left(U^{M}\right)^{\checkmark}\right)$ and this space is isomorphic in turn to $\boldsymbol{B}\left(U^{L}, U^{\bar{M}}\right)$. If we set $I\left(U^{L}, U^{M}\right)=\operatorname{dim} \operatorname{Hom}_{\mathscr{g}}\left(U^{L}, U^{M}\right)$, we have $I\left(U^{L}, U^{M}\right)=i\left(U^{L}, U^{\bar{M}}\right)$. Because $\bar{M}^{y}=\left(M^{y}\right)^{-}=\left(M^{y}\right)^{\complement}$, we have $I(L, M, x, y)=\operatorname{dim} \operatorname{Hom}_{G_{x}, y}\left(L^{x}, M^{y}\right)$ $=\operatorname{dim} B(L, \bar{M}, x, y)=i(L, \bar{M}, x, y)$ and this last number depends only on the $G_{1}: G_{2}$ double coset $a$ to which $x y^{-1}$ belongs, and may be written $I(L, M, a)$. Thus as an immediate consequence of Theorems 4 and 5 we have

THEOREM 6. Let $G_{1}$ and $G_{2}$ be closed regularly related subgroups of the separable locally compact group $\mathscr{G}$ such that there exist finite invariant measures on $\mathscr{G} / G_{1}$ and $\mathscr{G} / G_{2}$. Let $L$ and $M$ be continuous unitary representations of $G_{1}$ and $G_{2}$ respectively on separable Hilbert spaces. Let $\alpha$ be the image of Haar measure in the space $\mathscr{D}$ of $G_{1}: G_{2}$ double cosets and write $\alpha=\alpha_{1}+\alpha_{2}$ where $\alpha_{1}$ is a purely atomic measure and $\alpha_{2}$ is a nonatomic measure. If $I(L, M, a)>0$ on a measurable set of positive $\alpha_{2}$ measure, $I\left(U^{L}, U^{M}\right)=\infty$. If $I(L, M, a)=0, \alpha_{2}$ almost everywhere, then $I\left(U^{L}, U^{M}\right) \geqq \int I(L, M, a) d \alpha_{1}(a)$.

COROLLARY. Let $G$ be a closed subgroup of the separable locally compact group $\mathscr{G}$, suppose that $\mathscr{G} / G$ has a finite invariant measure and that $G$ is regularly related to itself. Let $\alpha$ be the image of Haar measure in the space $\mathscr{D}$ of $G: G$ 
double cosets and write $\alpha=\alpha_{1}+\alpha_{2}$ where $\alpha_{1}$ is a purely atomic measure and $\alpha_{2}$ is a nonatomic measure. Let $L$ be a continuous unitary representation of $G$. In order that $U^{L}$ be irreducible it is necessary that $I(L, L, a)=0, \alpha_{2}$ almost everywhere, and that $I(L, L, a) \leqq 1$ for all atoms $a \in \mathscr{D}$ and that $I(L, L, a)=1$ for at most one atom.

This corollary follows immediately from the theorem since $U^{L}$ is irreducible if and only if $I\left(U^{L}, U^{L}\right)=1$.

If $G$ is a closed normal subgroup then the space of $G: G$ double cosets is the quotient group $\mathscr{G} / G$; in particular, $G$ is regularly related to itself. The requirement that $\mathscr{G} / G$ have a finite invariant measure means exactly that $\mathscr{G} / G$ is compact. In this case the corollary states that for $U^{L}$ to be irreducible it is necessary that $L^{x}$ and $L$ be disjoint representations of $G$ for almost all $x \in \mathscr{G}$. However, Mackey [6] has found a much better result which states that if $L$ is irreducible, this condition is sufficient, at least in the case that $G$ is a type $I$ subgroup, regardless of the size of $\mathscr{G} / G$.

\section{REFERENCES}

1. N. Bourbaki, Intégration, Hermann, Paris, Chapters I-IV, 1952, Chapter V, 1956, Chapter VI, 1959.

2. F. Bruhat, Sur les représentations induites des groupes de Lie, Bull. Soc. Math. France 84 (1956), 97-205.

3. A. Grothendieck, Produits tensoriels topologiques et espaces nucléaires, Mem. Amer. Math. Soc. No. 16 (1956), $140 \mathrm{pp}$.

4. G. W. Mackey, Induced representations of locally compact groups. I, Ann. of Math. (2) 55 (1952), 101-139.

5. —_, Borel structure in groups and their duals, Trans. Amer. Math. Soc. 85 (1957), 134-165.

6. - Induced representations and normal subgroups, Proc. Internat. Sympos. Linear Spaces, Jerusalem, 1961.

7. C. C. Moore, On the Frobenius reciprocity theorem for locally compact groups, Pacific J. Math. 12 (1962), 359-365.

UNIVERSITY OF MARYLAND,

College Park, Maryland 